\title{
Article \\ Enhancement of the Luminescence Properties of Eu (III) Containing Paramagnetic Saponite Clays
}

\author{
Stefano Marchesi ${ }^{1}$ (D), Chiara Bisio ${ }^{1,2, *(\mathbb{D})}$ and Fabio Carniato ${ }^{1, *(\mathbb{D})}$ \\ 1 Dipartimento di Scienze e Innovazione Tecnologica, Università degli Studi del Piemonte Orientale “Amedeo \\ Avogadro", 15121 Alessandria, Italy; stefano.marchesi@uniupo.it \\ 2 CNR-SCITEC Istituto di Scienze e Tecnologie Chimiche "G. Natta”, Via C. Golgi 19, 20133 Milano, Italy \\ * Correspondence: chiara.bisio@uniupo.it (C.B.); fabio.carniato@uniupo.it (F.C.); Tel.: +39-013-136-0217 (F.C.)
}

Citation: Marchesi, S.; Bisio, C.; Carniato, F. Enhancement of the Luminescence Properties of Eu (III) Containing Paramagnetic Saponite Clays. Appl. Sci. 2021, 11, 8903. https://doi.org/10.3390/app11198903

Academic Editor: Francesco Enrichi

Received: 30 August 2021

Accepted: 21 September 2021

Published: 24 September 2021

Publisher's Note: MDPI stays neutral with regard to jurisdictional claims in published maps and institutional affiliations.

Copyright: (c) 2021 by the authors. Licensee MDPI, Basel, Switzerland. This article is an open access article distributed under the terms and conditions of the Creative Commons Attribution (CC BY) license (https:// creativecommons.org/licenses/by/ $4.0 /)$.
Abstract: In this study, 1,10-phenanthroline molecules were introduced in the interlayer space of a paramagnetic synthetic saponite clay containing both $\mathrm{Eu}^{3+}$ and $\mathrm{Gd}^{3+}$ ions in structural positions. Two samples with different loading of phenanthroline dye were prepared. The resulting hybrid materials possessed improved optical emission properties due to an efficient antenna effect from the phenanthroline to the $\mathrm{Eu}^{3+}$ centers; this effect was demonstrated to be higher than the metal-to-metal $\mathrm{Gd}^{3+}-\mathrm{Eu}^{3+}$ energy transfer previously studied. Insights on the relaxometric properties in aqueous solution of the solids after incorporation of the antenna groups were also obtained through NMR relaxometric analyses.

Keywords: europium; gadolinium; phenanthroline; clay; saponite; energy transfer; luminescence; relaxometry

\section{Introduction}

Following the definition of the International Union of Pure and Applied Chemistry (IUPAC), lanthanides are a unique series of 15 chemical elements with metallic properties, ranging from lanthanum (atomic number 57) to lutetium (71) [1,2]. They are historically known as rare-earth elements (REEs) along with the chemically similar scandium and yttrium elements [1].

Lanthanides are usually found in the trivalent oxidation state as ions $\left(\mathrm{Ln}^{3+}\right)$, while divalent $\left(\mathrm{Sm}^{2+}, \mathrm{Eu}^{2+}, \mathrm{Tm}^{2+}, \mathrm{Yb}^{2+}\right)$ and tetravalent $\left(\mathrm{Ce}^{4+}, \mathrm{Pr}^{4+}, \mathrm{Nd}^{4+}, \mathrm{Tb}^{4+}, \mathrm{Dy}^{4+}\right)$ forms are rarer [3-5]. As it is well-known, their chemistry is largely determined by the ionic radius which decreases along the series due to the lanthanide contraction effect [3]. The generic electron configuration for $\mathrm{Ln}^{3+}$ ions is [Xe] $4 f^{0-14}$, in which the electrons of $4 f$ orbitals are shielded from the external environment by outer-core $5 s^{2} 5 p^{6}$ sub-shells, which gives rise to a very abundant amount of electronic levels with well-defined energies [3]. This effect gives the lanthanides some very fascinating features, such as paramagnetic, luminescent and catalytic properties [6-8].

Indeed, the $4 f$ electron configurations determined several magnetic and electronic characteristics, such as considerable spin-orbit couplings and noteworthy paramagnetic character due to the unpaired electrons in $4 f$ orbitals (excluding $\mathrm{La}^{3+}$ and $\mathrm{Lu}^{3+}$ ) $[3,6]$. Moreover, magnetic moments, susceptibilities and anisotropy and electron relaxation times greatly differ through the series $[3,6]$. Owing to these properties, lanthanides were successfully employed in numerous magnetic applications: in the molecular magnetism as singlemolecule magnets (SMM) and for magnetic refrigeration [9-11]; in biomedical fields, as in the magnetic resonance imaging (MRI), with more than a few clinically-approved $\mathrm{Gd}^{3+}$ chelates contrast agents (CAs), or with $\mathrm{Gd}^{3+}$ (as ion or complex) embedded in nanosized biocompatible matrixes such as oxides, hydroxides, fluorides, oxysalts and silica based compounds [6,12-14]; and in novel superconductors, battery and REE-magnets [15,16]. 
Alongside their interesting magnetic features, lanthanides possessed very attractive optical properties and unique spectroscopic characteristics: sharp absorption bands with narrow and very recognizable emissions peaks of practically monochromatic radiations (in the NIR-UV-Vis range), long-lived excited state lifetimes and excellent photostability [17]. As a result of these unique features, many rare earth elements, i.e., $\mathrm{Eu}^{3+}, \mathrm{Tb}^{3+}, \mathrm{Er}^{3+}$, $\mathrm{Yb}^{3+}, \mathrm{Sm}^{3+}$, have been extensively used in numerous optical applications, from laboratory scale [18] to a more commercial/industrial level [16,19]. Notable applications include optical bioimaging (OI) and therapy [6,14,20,21]; energy-efficient and photonic devices such as phosphors for lighting and displays (i.e., light-emitting diodes (LEDs)), photoluminescent complexes and materials (i.e., $\beta$-diketones, metal-organic frameworks (MOFs)), luminescent sensors and probes (i.e., for NIR up-conversion), security inks and tags, photocatalysts, solar energy panels, fiber-optics and lasers [20-26]; luminescent nano-thermometry [27]; optical refrigeration [28] and quantum information processing [29].

The direct excitation of $4 f$ levels of luminescent $\mathrm{Ln}^{3+}$ ions generally produces a weak luminescence due to the parity-forbidden $f-f$ transitions which lead to weak light absorption (most molar absorption coefficients are $<10 \mathrm{~L} \cdot \mathrm{mol}^{-1} \cdot \mathrm{cm}^{-1}$ ) [3]. These drawbacks can be overcome by exploiting a process called sensitization, in which a chemical entity (donor group) can absorb and transfer energy to excited states of the $L n^{3+}$ ion (acceptor group), with a much increased enhancement of the resulting photoluminescence [30,31]. Different sensitization mechanisms have been studied over the years: (i) by adding another luminescent metal in close proximity to the $\mathrm{Ln}^{3+}$ (metal-to-metal energy transfer (MMET)) [32,33]; (ii) via charge-transfer states (i.e., metal-to-metal (MMCT), metal-to-ligand (MLCT) and ligand-to-metal (LMCT)) [34,35]; and (iii) by using specific organic ligands (chromophores) with intense absorption bands, which can funnel energy to the $L n^{3+}$ by an intramolecular energy transfer process (ligand-to-metal energy transfer (LMET), also called the antenna effect) $[36,37]$.

The development of novel magnetic/optical lanthanide systems represents an ongoing and exciting branch of research, especially through the encapsulation of lanthanides into inorganic materials, which can overcome the weakness of molecular complexes such as the weak thermal stability and low mechanical strength [38].

On this topic, in recent years, our research group has focused its attention on the combination of diverse paramagnetic and luminescent $f$-block elements with synthetic layered smectite clays. This class of materials represents an ideal host for the embedding of lanthanides, owing to its compelling physico-chemical characteristics such as high thermal stability, tuneable acidity, specific surface area, low production costs and outstanding chemical versatility $[39,40]$. Particularly, synthetic saponites can be easily modified to have different particle size and chemical composition of the lamellae and of the interlayer space, achieved by choosing specific synthetic one-pot and post-synthesis procedures [39]. Lanthanides such as $\mathrm{Gd}^{3+}, \mathrm{Eu}^{3+}$ and $\mathrm{Tb}^{3+}$ were included both in the form of complexes through intercalation procedures [41,42], and as ions by adding them in the clay synthesis gel [43-45], thus obtaining novel layered materials through a modified one-pot hydrothermal procedure in which the metal ions are directly placed in structural positions. This procedure, compared to post-synthesis intercalation, has the advantage to limit the production costs, reduce the synthesis times and increase the chemical stability of the modified material.

Among the saponite clays containing lanthanides, studied in the literature, the NaGdEuSAP, functionalized with both $\mathrm{Gd}^{3+}$ and $\mathrm{Eu}^{3+}$ ions in tetrahedral layers, exhibited noteworthy paramagnetic and photophysical properties, with the latter due to the existence of the $\mathrm{Gd}^{3+}-\mathrm{Eu}^{3+}$ energy transfer which led to an enhancement of the europium photoluminescence [45]. However, there is room for improvement on this topic, because the $L n^{3+}-L n^{3+}$ energy transfer is not the only possible sensitization route and the chemical composition of the clay interlamellar region can be modified through intercalation of chromophores that can interact with the $\mathrm{Eu}^{3+}$ and greatly enhance its optical properties. 
Following these ideas, in this work we investigated the enhancement of the luminescence of the $\mathrm{Eu}^{3+}$ centres in the $\mathrm{Na}-\mathrm{GdEuSAP}$ layered material, recently reported by our research group [45], with the use of an antenna group (1,10-phenanthroline, Phen), which was introduced in the interlayer space of the bifunctional synthetic saponite at two different loadings, with respect to the cation exchange capacity (C.E.C.) of the clay. Two final hybrid materials were prepared, hereafter called Phen-GdEuSAP_1 (lowest loading) and PhenGdEuSAP_2 (highest loading) (Scheme S1). Pristine $\mathrm{Na}^{+}$-exchanged saponite (Na-SAP) intercalated with Phen molecules was also prepared as the reference material (Phen-SAP). The 1,10-phenanthroline molecule was chosen because it is a common photo-enhancer for $\mathrm{Eu}^{3+}$-based compounds used in literature [46-49], since it is able to interact easily with $\mathrm{Eu}^{3+}$ and transfer energy to its nearby emitting sites through a ligand-to-metal energy transfer process (antenna effect) $[36,37,50]$. This non-radiative mechanism is based on a phononmediated coulombic (dipolar-dipolar) interaction between the Phen (donor group) and the $\mathrm{Eu}^{3+}$ (acceptor group), which can effectively enhance the photoluminescence performance of europium [46-49].

The final hybrid materials were submitted to a multi-technique physico-chemical characterization, with particular emphasis on the analyses of their photophysical properties and relaxometric features.

\section{Materials and Methods}

\subsection{Materials}

2.1.1. Synthesis of $\mathrm{Na}-\mathrm{SAP}$ Clay

Nanosized $\mathrm{Na}^{+}$-exchanged saponite clay with a cationic exchange capacity (CEC) of $87.9 \pm 2.3 \mathrm{meq} / 100 \mathrm{~g}$ was synthesized following the classical hydrothermal method [40]. In detail, $c a$. $6 \mathrm{~g}$ of $\mathrm{SiO}_{2}$ fumed were added to $29 \mathrm{~mL}$ of a basic solution containing $0.465 \mathrm{~g}$ of $\mathrm{NaOH}$. After $1 \mathrm{~h}, 18.8 \mathrm{~g}$ of magnesium acetate tetrahydrate, $2.35 \mathrm{~g}$ of aluminium isopropoxide and $8.2 \mathrm{~mL}$ of water were added to the previous suspension. The gel was stirred for $2 \mathrm{~h}$, transferred into an autoclave and heated at $240^{\circ} \mathrm{C}$ for $72 \mathrm{~h}$. The final sample (SAP-20) was purified with water. Finally, $1 \mathrm{~g}$ of SAP-20 was dispersed in a saturated $\mathrm{NaCl}$ solution $(100 \mathrm{~mL})$ for $36 \mathrm{~h}$ at $25^{\circ} \mathrm{C}$. The solid (Na-SAP-20) was filtered and washed with water.

\subsubsection{Synthesis of Na-GdEuSAP Clay}

The synthetic saponite clay containing both $\mathrm{Gd}^{3+}$ and $\mathrm{Eu}^{3+}$ ions in structural position of the inorganic framework was prepared following the one-pot hydrothermal procedure recently reported in the literature by our research group [45].

\subsubsection{Intercalation of 1,10 -Phenathroline in Saponite Clays}

Phen-GdEuSAP_ $n(n=1,2)$ samples were prepared through intercalation of 1,10 phenanthroline (Phen) in a molar ratio of 1 and 2 times with respect to the cation exchange capacity (C.E.C.) of Na-GdEuSAP clay (Scheme S1). In detail, an appropriate amount of Phen was dissolved in $35 \mathrm{~mL}$ of ultrapure water at $\mathrm{pH}$ between 4 and 5 , then $0.35 \mathrm{~g}$ of $\mathrm{Na}-\mathrm{GdEuSAP}$ were added and the mixture was stirred at room temperature (RT) for $6 \mathrm{~h}$. After that, the suspension was centrifugated ( $8000 \mathrm{rpm}$ for $5 \mathrm{~min}$ ) and the solid was washed with ethanol $(\mathrm{EtOH}, 5 \times 50 \mathrm{~mL})$ and hot ultrapure water $(5 \times 50 \mathrm{~mL})$. The solid was finally dried in an oven overnight at $100{ }^{\circ} \mathrm{C}$.

Reference materials were obtained by intercalation of Phen molecules in Na-SAP clays following the same procedure described before. The samples were named Phen-SAP $n$, where $n=1,2$.

\subsection{Analytical Methods}

The cationic exchange capacity (C.E.C.) parameter of Na-GdEuSAP clay was determined by the Ultraviolet-Visible (UV-Vis) method reported here: $0.300 \mathrm{~g}$ of clay were exchanged with $10 \mathrm{~mL}$ of a $0.02 \mathrm{M}$ solution of hexamminecobalt(III) chloride $\left(\left[\mathrm{Co}\left(\mathrm{NH}_{3}\right)_{6}\right]^{3+}\right)$ 
at RT for $60 \mathrm{~h}$. After separation by centrifugation (5000 rpm for $5 \mathrm{~min}$, two times), the solution was analyzed by UV-Vis spectroscopy. UV-Visible spectra were recorded at RT in the range 300-600 $\mathrm{nm}$ with a resolution of $1 \mathrm{~nm}$, using a double-beam Perkin Elmer Lambda 900 Spectrophotometer (Perkin Elmer, Waltham, MA, USA). The absorbance of the band at $475 \mathrm{~nm}\left({ }^{1} \mathrm{~A}_{1 \mathrm{~g}} \rightarrow{ }^{1} \mathrm{~T}_{1 \mathrm{~g}}\right)$, relative to a $d$ - $d$ spin-allowed Laporte-forbidden transition of $\mathrm{Co}^{3+}$, was evaluated to quantify the amount of free $\mathrm{Co}^{3+}$ ions in solution, thereby determining the amount exchanged in the procedure and thus the C.E.C. of the saponite sample.

The elemental analyses were performed on a Thermo Fisher Scientific X5 Series Inductively Coupled Plasma Mass Spectrometer (ICP-MS; Waltham, MA, USA). Prior to the analyses, the solids were mineralized by treatment with a mixture of nitric acid $\left(\mathrm{HNO}_{3}\right.$, $5 \mathrm{~mL}$ ) and hydrofluoric acid (HF, $5 \mathrm{~mL}$ ) at $100{ }^{\circ} \mathrm{C}$ for $8 \mathrm{~h}$. The samples were opportunely diluted in nitric acid aqueous solution (1 wt $\%$ ) before the analysis.

$X$-ray powder (XRPD) diffractograms were collected on unoriented ground powders with a ThermoARL X'TRA-048 Powder Diffractometer (Thermo Fisher Scientific, Waltham, MA, USA) with a Cu-K $\alpha 1(\lambda=1.54062 \AA)$ monochromatic radiation. Diffractograms were recorded at room temperature (RT) in the $2^{\circ}-65^{\circ} 2 \theta$ range with a step size of $0.02^{\circ}$ and a rate of $1.0^{\circ} / \mathrm{min}$. The X-ray profiles at low angles $\left(2-15^{\circ} 2 \theta\right)$ were collected with narrower slits and rate of $0.25^{\circ} / \mathrm{min}$.

Thermogravimetric analyses (TGA) were carried out with a Setaram SETSYS Evolution Thermobalance. The data were collected in the range of 50 to $900{ }^{\circ} \mathrm{C}$, with a scan rate of $10{ }^{\circ} \mathrm{C} / \mathrm{min}$ and under oxygen $\left(\mathrm{O}_{2}\right)$ flow $(100 \mathrm{~mL} / \mathrm{min})$.

Photoexcitation and photoluminescence (PL) emission spectra were recorded on a Horiba Jobin-Yvon Model IBH FL-322 Fluorolog 3 Spectrometer equipped with a $450 \mathrm{~W}$ xenon arc lamp, double grating excitation and emission monochromators $\left(2.1 \mathrm{~nm} \cdot \mathrm{mm}^{-1}\right.$ dispersion; 1200 grooves per mm) and a Hamamatsu Model R928 photomultiplier tube. Time-resolved measurements were performed by using the time-correlated single-photon counting (TCSPC) option. A $295 \mathrm{~nm}$ nanoLED laser was used to excite the samples. Signals were collected using an IBH DataStation Hub photon counting module. Data analysis was performed using the commercially available DAS6 software (HORIBA Jobin Yvon IBH).

The water proton longitudinal relaxation rates $\left(R_{1}\right)$ were measured by using a variable field relaxometer equipped with an HTS-110 3T Metrology Cryogen-free Superconducting Magnet (Mede, Italy), operating in the overall range of proton Larmor frequencies of 20-120 MHz (0.47-3.00 T). The measurements were performed using the standard inversion recovery sequence (20 experiments, 2 scans) with a typical $90^{\circ}$ pulse width of $3.5 \mu$ s and the reproducibility of the data was within $\pm 0.5 \%$. The temperature was controlled with a Stelar VTC-91 heater airflow equipped with a copper-constantan thermocouple (uncertainty of $\pm 0.1^{\circ} \mathrm{C}$ ). Additional points in the $0.01-10 \mathrm{MHz}$ frequency range were collected on a Fast-Field Cycling (FFC) Stelar SmarTracer Relaxometer. Na-GdEuSAP, Phen-GdEuSAP_1 and Phen-GdEuSAP_2 solids $(10 \mathrm{mg})$ were dispersed in $1.5 \mathrm{~mL}$ of ultrapure water, in the presence of xanthan gum $(0.1 \mathrm{wt} . \%)$ to improve particle dispersion. Before measurements, the suspensions were sonicated for $30 \mathrm{~min}$. The $\mathrm{pH}$ of the suspensions was ca. 7.0.

\section{Results and Discussion}

As reported in the experimental section, Phen-GdEuSAP_ $n$ materials $(n=1,2)$ were prepared by the intercalation process of 1,10-phenanthroline (Phen) in the interlayer space of $\mathrm{Na}-\mathrm{GdEuSAP}$ clay, recently studied in the literature by our research group [45]. Since Phen was intercalated in acidic pH conditions (between 4 and 5), below its pKa (equal to 4.8 at $298 \mathrm{~K}$ ) [51], the nitrogen atoms of the molecule are protonated and then suitable for the cation exchange process. Phen was added in a molar quantity equal to 1 and 2 times with respect to the cation exchange capacity of Na-GdEuSAP (C.E.C. $=44.7 \pm 7.9 \mathrm{meq} / 100 \mathrm{~g}$ ) (see Figure S1) [45], in order to have a sufficient amount of the chromophore in close proximity to the structural europium sites, thus ensuring an efficient energy transfer from the antenna group to $\mathrm{Eu}^{3+}$. The same procedure was adopted for the preparation of the reference Phen-SAP. The $\mathrm{Gd}^{3+}$ and $\mathrm{Eu}^{3+}$ contents in the Na-GdEuSAP, determined by 
inductively coupled plasma mass spectrometry (ICP-MS), were previously found to be $0.026 \mathrm{mmol} / \mathrm{g}$ for both metals [45].

The X-ray powder diffraction (XRPD) analyses show that the typical crystallographic reflections of the saponite clay are maintained after the intercalation procedure (Figure 1A,B) $[39,40]$. Furthermore, the analyses suggested the intercalation of the Phen in the Na-GdEuSAP, as indicated by the marked shift of the basal plane (001) reflection in the modified clays with respect to un-modified Na-GdEuSAP, from $7.3^{\circ} 2 \theta(1.21 \mathrm{~nm})$ to $6.40^{\circ}$ $2 \theta(1.38 \mathrm{~nm})$ (Figure 1B). No traces of well resolved peaks associated to the crystalline Phen (Figure S2) were detected in XRD patterns of the hybrid materials, thus demonstrating the absence of extra-phase Phen species.
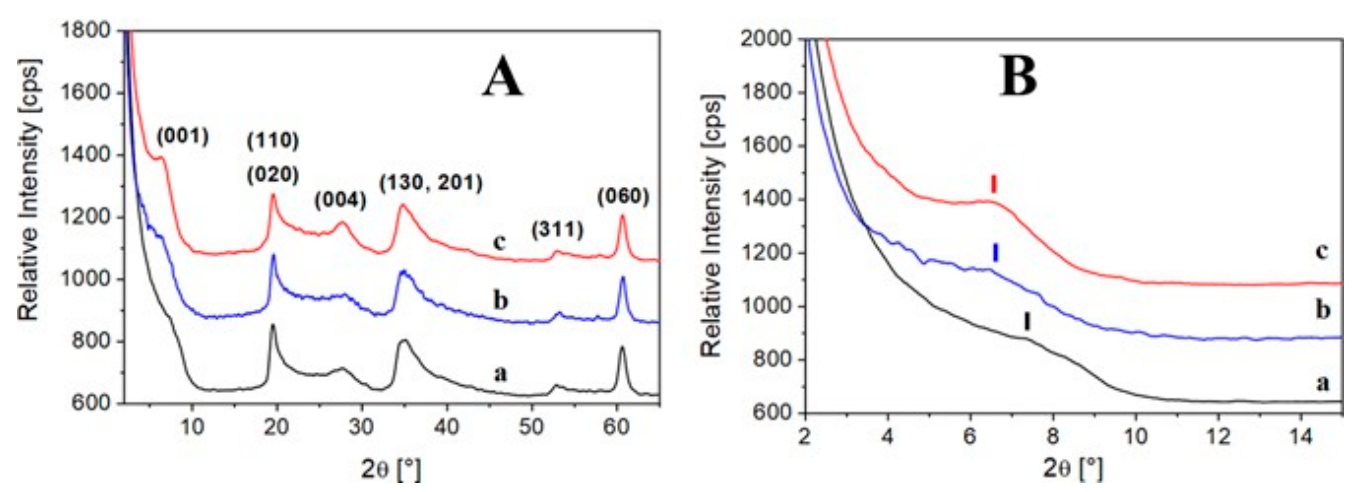

Figure 1. (A) X-ray powder diffraction profiles of Na-GdEuSAP (a), Phen-GdEuSAP_1 (b) and Phen-GdEuSAP_2 (c). Diffractograms collected at low angles $\left(2^{\circ}-15^{\circ} 2 \theta\right)$ are reported in $(\mathbf{B})$.

Thermogravimetric analyses (TGA) were performed to (i) further confirm the intercalation of the Phen in the Na-GdEuSAP clay, (ii) evaluate its thermal stability in the hybrid materials and (iii) quantify the amount of Phen confined in the clays. The TGA profile of Na-GdEuSAP, collected under oxygen flow, shows a first weight loss at $100{ }^{\circ} \mathrm{C}$ due to the presence of physisorbed water and a second one in the $150-750{ }^{\circ} \mathrm{C}$ range, associated to a gradual release of interlamellar water and the initial dehydroxylation of the layered structure (Figure 2A). The thermal profiles of the intercalated saponites are mainly characterized by the presence of a relevant weight loss in the $430-560^{\circ} \mathrm{C}$ range, attributed to the degradation of the Phen molecules (Figure 2B). The degradation of the intercalated molecules appears delayed by $c a .100^{\circ} \mathrm{C}$ with respect to the pure compound analyzed in the same conditions (Figure S3). The increase in the thermal stability of the Phen in PhenGdEuSAP samples confirms its presence in the interlayer space [41,42]. The intercalated amount of Phen, expressed as mmol of Phen per gram of saponite, was extrapolated from the TGA curves (Figure 2A) and the results are reported in Table 1. The Phen-GdEuSAP_1 contains $0.29 \mathrm{mmol} / \mathrm{g}$ of Phen, lower than that of the C.E.C. of the clay $(0.447 \mathrm{mmol} / \mathrm{g})$, suggesting that the interlayer space is partially occupied by the organic molecules. For Phen-GdEuSAP_2, the amount of Phen was $0.50 \mathrm{mmol} / \mathrm{g}$, comparable to the C.E.C. of the clay.

The photophysical properties of Phen-GdEuSAP samples were thoroughly investigated by photoluminescence spectroscopy $(\mathrm{PL})$ in order to $(i)$ evaluate the occurrence of Phen $\rightarrow \mathrm{Eu}^{3+}$ energy transfer and the related optical enhancement of $\mathrm{Eu}^{3+}$ centers, (ii) obtain information on the dependence of the energy transfer on the Phen loading and (iii) determine the parameters that define the energy transfer mechanism $[36,37,50]$. The Phen acted as an energy donor species/antenna group (donor, D) to the europium ion (acceptor, A), leading to the formation of a 'D-A complex' that greatly enhances the photoluminescence properties of europium. $\mathrm{Na}^{+}$-exchanged saponites intercalated with Phen molecules were also studies as reference materials. All the samples were studied at the solid state. 

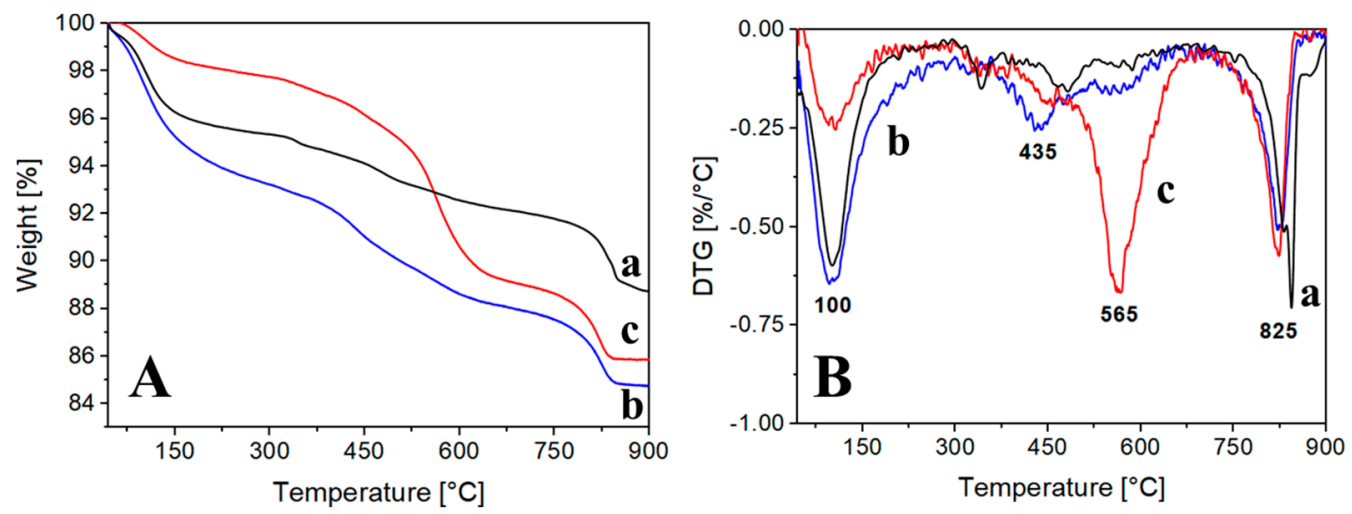

Figure 2. TGA (A) and DTG (B) profiles of Na-GdEuSAP (a), Phen-GdEuSAP_1 (b) and PhenGdEuSAP_2 (c). The analyses were carried out under oxygen flow $(100 \mathrm{~mL} / \mathrm{min})$.

Table 1. Intercalated amount of Phen [mmol/g] in Phen-GdEuSAP_1 and Phen-GdEuSAP_2.

\begin{tabular}{cc}
\hline Sample & $\begin{array}{c}\text { Phen Content } \\
{[\mathbf{m m o l} / \mathrm{g}]}\end{array}$ \\
\hline Phen-GdEuSAP_1 & 0.29 \\
Phen-GdEuSAP_2 & 0.50 \\
\hline
\end{tabular}

The excitation spectrum of the Phen-GdEuSAP_1 sample collected at $615 \mathrm{~nm}$ (Figure S4) shows the characteristic narrow peaks of the intra- $4 f^{6}$ electronic transitions of $\mathrm{Eu}^{3+}\left({ }^{5} \mathrm{D}_{0}{ }^{7} \mathrm{~F}_{J}\right.$, $\left.J=0-4 ; \lambda_{\max }=395 \mathrm{~nm},{ }^{7} \mathrm{~F}_{0}{ }^{-5} \mathrm{~L}_{6}\right)$ [52] and $\mathrm{Gd}^{3+}\left({ }^{8} \mathrm{~S}_{7 / 2}{ }^{6}{ }^{6} \mathrm{I}_{\mathrm{J}} ; \lambda_{\max }=272 \mathrm{~nm},{ }^{8} \mathrm{~S}_{7 / 2}{ }^{6} \mathrm{P}_{\mathrm{J}}\right)$ [53] together with the $S_{0} \rightarrow S_{1}\left({ }^{1} \pi \rightarrow{ }^{1} \pi^{*}\right)$ electronic transition of the Phen, located at $290 \mathrm{~nm}[46-49,54]$ (Figure S5A).

The emission spectra of both Phen-GdEuSAP samples at solid state upon direct excitation of $\mathrm{Eu}^{3+}$ at $\lambda_{\max }=395 \mathrm{~nm}$ were found to be comparable with that of the starting Na-GdEuSAP clay measured in the same experimental conditions (Figure S6) [45].

The emission spectra of Phen-GdEuSAP samples, collected under irradiation at both $290\left(\lambda_{\mathrm{exc}}\right.$ of Phen) and $395 \mathrm{~nm}\left(\lambda_{\mathrm{exc}}\right.$ of $\left.\mathrm{Eu}^{3+}\right)$ (Figure 3), showed that upon direct excitation of the antenna group there is a marked increase in the intensity of the hypersensitive ${ }^{5} \mathrm{D}_{0}{ }^{7} \mathrm{~F}_{2}$ transition at $615 \mathrm{~nm}$ of Eu${ }^{3+}$ compared to the direct excitation of the lanthanide, thus confirming the interaction of Phen molecules with $\mathrm{Eu}^{3+}$ sites and the occurrence of the ligand-to-metal energy transfer phenomenon $[36,37,50]$. The emission spectra of both hybrid materials excited at $290 \mathrm{~nm}$ ( $\lambda_{\max }$ of Phen) shown in Figure 3 also show a broad emission band centered at $415 \mathrm{~nm}$ associated with the residual $\mathrm{T}_{1} \rightarrow \mathrm{S}_{0}\left({ }^{3} \pi^{*} \rightarrow{ }^{1} \pi\right)$ electronic transition of Phen [46-49,54] (Figure S5B).
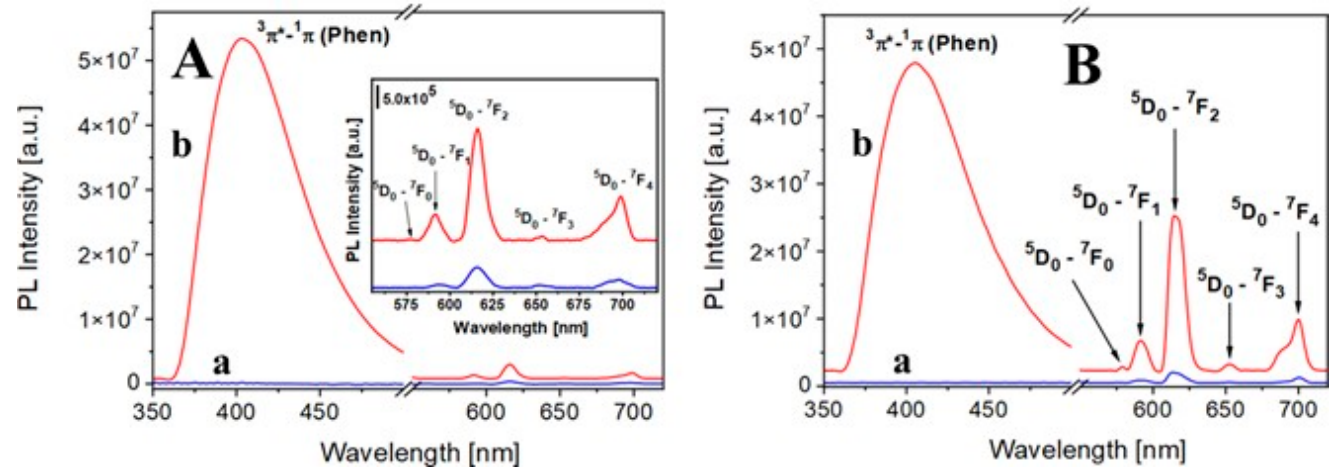

Figure 3. Emission spectra at solid state of Phen-GdEuSAP_1 (A) and Phen-GdEuSAP_2 (B), collected under excitation at $395\left(\lambda_{\max }\right.$ of $\left.\mathrm{Eu}^{3+},(\mathrm{a})\right)$ and $290 \mathrm{~nm}\left(\lambda_{\max }\right.$ of Phen, (b)), in the 350-720 nm emission range. 
The best optical improvement under the energy transfer condition, related to the sensitization of the intensity emission of the $615 \mathrm{~nm}$ band of europium, was observed for the Phen-GdEuSAP_2 sample, with an increase of $+1051 \%$ (Figure 3B $(b)$ ) compared to the direct excitation of europium at $395 \mathrm{~nm}$ (Figure 3B(a)). This enhancement is considerably higher than the value observed by exploiting the $\mathrm{Gd}^{3+} \rightarrow \mathrm{Eu}^{3+}$ energy transfer mechanism $(+240 \%)$ in the Na-GdEuSAP clay at solid state [45].

Moreover, $\mathrm{Eu}^{3+}$ centers located in the inorganic framework experienced a local low symmetry environment after the intercalation of Phen molecules. The asymmetry factor (R) [55-58] for the two intercalated samples is in the 5 to 6 range, higher than that calculated for Na-GdEuSAP $(\mathrm{R}=3.10)$. The $\mathrm{R}$ parameter was extrapolated from the intensity ratio of electric-dipole ${ }^{5} \mathrm{D}_{0} \rightarrow{ }^{7} \mathrm{~F}_{2}$ on magnetic-dipole ${ }^{5} \mathrm{D}_{0} \rightarrow{ }^{7} \mathrm{~F}_{1}$ transitions in the emission spectra (Figure 3).

An evaluation of the colorimetric features of Phen-GdEuSAP clays was also carried out by calculating the chromaticity coordinates $(x y)$ and related RGB and Hex parameters according to CIE 1931 color spaces [59]. The chromaticity diagrams were extrapolated from the emission spectra collected at solid state of Phen-GdEuSAP samples shown in Figure 3. As reported in Figure 4, and from the calculated parameters of Table S1, the Phen-to-Eu ${ }^{3+}$ energy transfer process led to final emissions in the blueish-violet visible region of the electromagnetic spectrum (b), especially visible for Phen-GdEuSAP_2, compared to the pure red emission of the direct excitation of $\mathrm{Eu}^{3+}(\mathrm{a})$. Accordingly, it was found that is possible to modulate the emission color of the entire system by changing the amount of antenna groups introduced in the material.
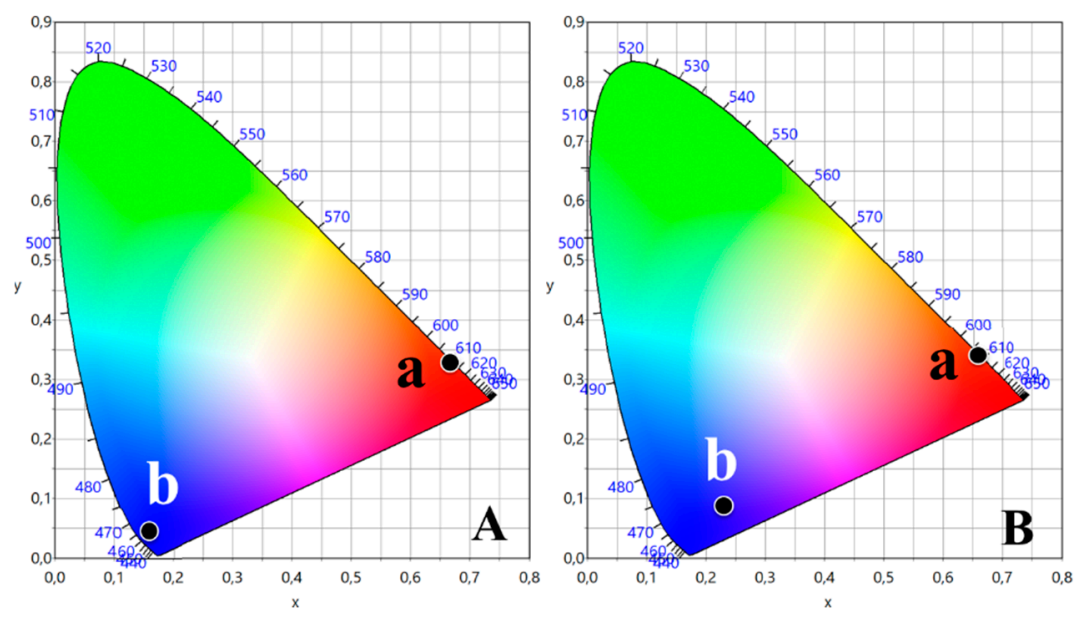

Figure 4. CIE $1931 x y$ chromaticity diagrams of Phen-GdEuSAP_1 (A) and Phen-GdEuSAP_2 (B) samples $\left(\lambda_{\text {exc }}\right.$ of 395 (a) and $290 \mathrm{~nm}$ (b)), derived from emission spectra reported in Figure 3.

The results obtained from the photoluminescence measurements were further confirmed by the analysis of the parameters that describe the efficiency $\left(E_{\mathrm{EnT}}\right)$ and the constant rate $\left(k_{\mathrm{EnT}}\right)$ of the energy transfer process between the ligand and the lanthanide ions, as presented in Equations (1) and (2). [36,37,50,60,61]:

$$
\begin{aligned}
& E_{E n T}[\%]=\left(1-\frac{\tau_{D A}}{\tau_{D}}\right) \cdot 100 \\
& k_{E n T}\left[s^{-1}\right]=\left(\frac{1}{\tau_{D A}}-\frac{1}{\tau_{D}}\right)
\end{aligned}
$$

The parameters were derived from the time-resolved fluorescence spectroscopy analyses at solid state of the experimental lifetimes $\left(\tau_{\mathrm{D}}\right)$ of the Donor system (Phen-SAP clays) and those $\left(\tau_{\mathrm{DA}}\right)$ of the Donor-Acceptor (Phen-GdEuSAP clays), collecting the decay curves of the $S_{0}$ excited state of Phen at $415 \mathrm{~nm}[46-49,54]$ upon excitation at $295 \mathrm{~nm}$ (Figure S7A,B for Phen-GdEuSAP samples and Figure S8A,B for Phen-SAP samples). The curves were 
well fitted with a bi-exponential function, obtaining average $\tau$ values for each one. The results, reported in Table 2, show that Phen-GdEuSAP_2 is characterized by the most efficient optical communication between Phen molecules and $\mathrm{Eu}^{3+}$ centers, with the highest energy transfer efficiency of $c a .21 \%$ and a rate constant of $6.31 \times 10^{7} \mathrm{~s}^{-1}$. The values of the calculated rate constants suggest that the transfer process from Phen to the $\mathrm{Eu}^{3+}$ sites occurs following the Förster resonance energy transfer (FRET) mechanism.

Table 2. Experimental lifetimes $(\tau)$ at solid state of Phen-SAP (D) and Phen-GdEuSAP (DA) samples $\left(1=\right.$ Phen-GdEuSAP_1; $2=$ Phen-GdEuSAP_2) under excitation at $295 \mathrm{~nm}$; efficiency $\left(E_{\text {EnT }}\right)$ and rate constant $\left(k_{\mathrm{EnT}}\right)$ parameters of the Phen $\rightarrow \mathrm{Eu}^{3+}$ FRET mechanism.

\begin{tabular}{ccc}
\hline Sample & $\mathbf{1}$ & $\mathbf{2}$ \\
\hline$\tau_{\mathrm{D}}[\mathrm{ns}]$ & 3.82 & 4.28 \\
$\tau_{\mathrm{DA}}[\mathrm{ns}]$ & 3.41 & 3.37 \\
$k_{\mathrm{EnT}}\left[\mathrm{s}^{-1}\right]$ & $3.15 \times 10^{7}$ & $6.31 \times 10^{7}$ \\
$E_{\mathrm{EnT}}[\%]$ & 10.73 & 21.26 \\
\hline
\end{tabular}

The lifetime of the excited states of $\mathrm{Eu}^{3+}$ for $\mathrm{Na}-\mathrm{GdEuSAP}$ and the solids containing different amounts of 1,10-phenanthroline were also measured by irradiating the samples at $370 \mathrm{~nm}$ and monitoring the electronic transition at $615 \mathrm{~nm}$. A gradual increase of the decay lifetime of $\mathrm{Eu}^{3+}$ from $0.05 \mathrm{~ms}$ for Na-GdEuSAP to 0.11 and $0.28 \mathrm{~ms}$ for PhenGdEuSAP_1 and Phen-GdEuSAP_2, respectively, was detected (Figure S9). This could suggest a stabilization of the excited state of $\mathrm{Eu}^{3+}$ ions, promoted by the presence of the 1,10-phenanthroline molecules.

Finally, the aqueous suspension of Phen-GdEuSAP_1 and Phen-GdEuSAP_2 clays were submitted to ${ }^{1} \mathrm{H}-\mathrm{NMR}$ relaxometric analysis by measuring the $1 / T_{1}{ }^{1} \mathrm{H}$ nuclear magnetic dispersion (NMRD) profiles, collected in the magnetic field range of $0.01-120 \mathrm{MHz}$ at $37^{\circ} \mathrm{C}$ and neutral $\mathrm{pH}$ (Figure 5). The samples were measured in aqueous suspension, in the presence of $0.1 \mathrm{wt} . \%$ of xanthan gum in order to improve the suspension stability over time. The relaxivity parameter, expressed as $r_{1},\left[\mathrm{mM}^{-1} \cdot \mathrm{s}^{-1}\right]$, was measured as a function of the applied magnetic field strength $[41,42,62,63]$, by evaluating the relaxation rate of bulk water protons $\left(R_{1}\right)$ in the presence of the paramagnetic entity and dividing the obtained value by the concentration (expressed in $\mathrm{mM}$ ) of $\mathrm{Gd}^{3+}$ centers.

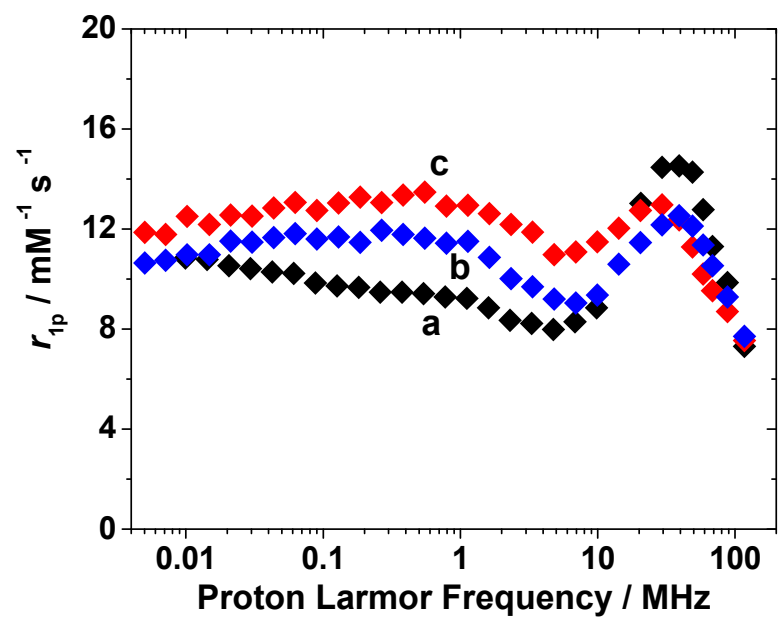

Figure 5. $1 / \mathrm{T}_{1}{ }^{1} \mathrm{H}-\mathrm{NMRD}$ profiles of Na-GdEuSAP (a), Phen-GdEuSAP_1 (b) and Phen-GdEuSAP_2 (c) at $37^{\circ} \mathrm{C}$, over the frequency range $0.01-120 \mathrm{MHz}$ and neutral $\mathrm{pH}$.

The profiles of Phen-GdEuSAP_1 (b) and Phen-GdEuSAP_2 (c) exhibited a similar shape compared to that of Na-GdEuSAP measured in the previous study (Figure 5) [45]. This indicates that the relaxometric properties remain almost unchanged after introduction 
of Phen in the interlamellar region of the clay. Furthermore, this also suggests that Phen molecules present in the interlayer space are probably not directly coordinated to the lanthanide centers of the inorganic structure, but they actually reside in close proximity to them, promoting the energy transfer process to $\mathrm{Eu}^{3+}$ observed in the fluorescence analyses Figure S4 and Figure 3), without dramatically changing the paramagnetic properties generated by the structural $\mathrm{Gd}^{3+}$ ions.

\section{Conclusions}

In this work, two hybrid materials were prepared through intercalation of 1,10phenanthroline molecules in a bifunctional optical/paramagnetic saponite clay containing $\mathrm{Eu}^{3+}$ and $\mathrm{Gd}^{3+}$ ions in structural positions. The phenanthroline was introduced in the interlayer space of the saponite at two different loadings. The hybrid materials, which already possessed interesting optical and magnetic properties, exhibit significantly improved luminescent features upon interaction of phenanthroline molecules with $\mathrm{Eu}^{3+}$ ions, especially in the sample with the highest organic loading, due to an efficient antenna effect from the ligand to the metallic centers, which is even greater than the $\mathrm{Gd}^{3+}-\mathrm{Eu}^{3+}$ energy transfer previously observed. From a magnetic point of view, the solids maintain similar relaxometric properties in aqueous solution compared to the starting bifunctional saponite. The properties displayed by the hybrid materials prepared in this study make them desirable in applications such as optical and MRI probes or as light-emitting devices.

Supplementary Materials: The following are available online at https: / / www.mdpi.com/article/10.3 390/app11198903/s1, Scheme S1: Schematic view of the intercalation procedure of 1,10-phenanthroline (Phen) molecules into the interlayer space of Na-GdEuSAP, at two different loadings with respect to the cation exchange capacity of the clay, and of the simplified structure of the final material; Figure S1. (A) UV-Vis spectra of $\left[\mathrm{Co}\left(\mathrm{NH}_{3}\right)_{6}\right]^{3+}$ standard solutions at $25^{\circ} \mathrm{C}$ with concentration in the $0.05-0.005 \mathrm{M}$ range. (B) Calibration curve extrapolated by the UV-Vis spectra $\left(\lambda_{\mathrm{abs}}=475 \mathrm{~nm}\right)$. (C) UV-Vis spectra of the supernatant of Na-GdEuSAP, obtained after treatment of the solid in $0.02 \mathrm{M}$ $\left[\mathrm{Co}\left(\mathrm{NH}_{3}\right)_{6}\right]^{3+}$ at $25^{\circ} \mathrm{C}$ for $60 \mathrm{~h}$; Figure S2: X-ray profile of crystalline Phen; Figure S3: TGA (A) and DTG (B) profiles of crystalline Phen. The analyses were carried out under oxygen flow $(100 \mathrm{~mL} / \mathrm{min})$; Figure S4: Excitation spectrum at solid state of Phen-GdEuSAP_1, monitored at $615 \mathrm{~nm}$; Figure S5: (A) Excitation spectrum at solid state of Phen, monitored at $415 \mathrm{~nm}$. (B) Emission spectrum at solid state of Phen, under excitation at $290 \mathrm{~nm}$; Figure S6: Emission spectra at solid state of Na-GdEuSAP (a) [1], Phen-GdEuSAP_1 (b) and Phen-GdEuSAP_2 (c), under excitation at 395 nm; Figure S7: PL T 1 -S 0 (415 $\mathrm{nm}$ ) intensity decay over time of Phen-GdEuSAP_1 (A) and Phen-GdEuSAP_2 (B) under irradiation at $295 \mathrm{~nm}$ at solid state. The curves fitting was performed with a bi-exponential function (black lines). The $\chi^{2}$ and RSS (residual sum of squares) values are reported in the table below; Figure S8: PL T $1-S_{0}$ $(415 \mathrm{~nm})$ intensity decay over time of Phen-SAP_1 (A) and Phen-SAP_2 (B), under irradiation at 295 $\mathrm{nm}$ at solid state. The curve fitting was performed with a bi-exponential function (black lines). The $\chi^{2}$ and RSS (residual sum of squares) values are reported in the table below; Figure S9. PL ${ }^{5} \mathrm{D}_{0}-{ }^{7} \mathrm{~F}_{2}(615$ nm) intensity decay over time of Na-GdEuSAP (A), Phen-GdEuSAP_1 (B) and Phen-GdEuSAP_2 (C), under irradiation at $370 \mathrm{~nm}$ at solid state. The curve fitting was performed with a bi-exponential function (black lines); Table S1: Photometric data, in accordance with CIE 1931 colour spaces, for Phen-GdEuSAP_1 and Phen-GdEuSAP_2, extrapolated from emission spectra at solid state shown in Figure 3.

Author Contributions: Conceptualization, S.M., C.B. and F.C.; methodology, S.M., C.B. and F.C.; formal analysis, S.M.; investigation, S.M.; data curation, S.M. and F.C.; writing-original draft preparation, S.M.; writing-review and editing, C.B. and F.C. All authors have read and agreed to the published version of the manuscript.

Funding: Financial support from the Università del Piemonte Orientale (FAR-2019) is acknowledged.

Institutional Review Board Statement: Not applicable.

Informed Consent Statement: Not applicable.

Data Availability Statement: Not applicable. 
Acknowledgments: The authors are fully grateful to Elena Perin (DiSIT, Università del Piemonte Orientale, Alessandria, Italy) for the ICP-MS analyses.

Conflicts of Interest: The authors declare no conflict of interest.

\section{References}

1. Balaram, V. Rare earth elements: A review of applications, occurrence, exploration, analysis, recycling, and environmental impact. Geosci. Front. 2019, 10, 1285-1303. [CrossRef]

2. Liddle, S.T. International Year of the Periodic Table: Lanthanide and Actinide Chemistry. Angew. Chem. Int. Ed. 2019, 58, 5140-5141. [CrossRef]

3. Housecroft, C.E.; Sharpe, A.G. Chapter 27-The f-block metals: Lanthanoids and actinoids. In Inorganic Chemistry, 5th ed.; Mo, X., Santosh, M., Wan, X., Eds.; Pearson Education UK: London, UK, 2018; pp. 1033-1064.

4. Nicholas, H.M.; Mills, D.P. Lanthanides: Divalent Organometallic Chemistry. Encycl. Inorg. Bioinorg. Chem. 2017, 1-10. [CrossRef]

5. Gompa, T.P.; Ramanathan, A.; Rice, N.T.; La Pierre, H.S. The chemical and physical properties of tetravalent lanthanides: Pr, Nd, Tb, and Dy. Dalton Trans. 2020, 49, 15945-15987. [CrossRef] [PubMed]

6. $\quad$ Dong, H.; Du, S.-R.; Zheng, X.-Y.; Luy, G.-M.; Sun, L.-D.; Li, L.-D.; Zhang, P.-Z.; Zhang, C.; Yan, C.-H. Lanthanide Nano-particles: From Design toward Bioimaging and Therapy. Chem. Rev. 2015, 115, 10725-10815. [CrossRef] [PubMed]

7. Bünzli, J.-C.G. Rising stars in science and technology: Luminescent lanthanide materials. Eur. J. Inorg. Chem. 2017, 2017, 5058-5063. [CrossRef]

8. Hernández, W.; Laguna, O.; Centeno, M.A.; Odriozola, J.A. Structural and catalytic properties of lanthanide (La, Eu, Gd) doped ceria. J. Solid State Chem. 2011, 184, 3014-3020. [CrossRef]

9. Zhu, Z.; Guo, M.; Li, X.-L.; Tang, J. Molecular magnetism of lanthanide: Advances and perspectives. Coord. Chem. Rev. 2019, 378, 350-364. [CrossRef]

10. Marin, R.; Brunet, G.; Murugesu, M. Shining New Light on Multifunctional Lanthanide Single-Molecule Magnets. Angezw. Chem. Int. Ed. 2021, 60, 1728-1746. [CrossRef]

11. Yu, H.; Yang, J.-X.; Han, J.-Q.; Li, P.-F.; Hou, Y.-L.; Wanf, W.-M.; Fang, M. Tetranuclear lanthanide complexes showing magnetic refrigeration and single molecule magnet behaviour. New J. Chem. 2019, 43, 8067-8074. [CrossRef]

12. Wahsner, J.; Gale, E.M.; Rodríguez-Rodríguez, A.; Caravan, P. Chemistry of MRI Contrast Agents: Current Challenges and New Frontiers. Chem. Rev. 2019, 119, 957-1057. [CrossRef] [PubMed]

13. Carniato, F.; Tei, L.; Botta, M. Gd-Based Mesoporous Silica Nanoparticles as MRI Probes. Eur. J. Inorg. Chem. 2018, 2018, 4936-4954. [CrossRef]

14. Amoroso, A.J.; Pope, J.A. Using lanthanide ions in molecular bioimaging. Chem. Soc. Rev. 2015, 44, 4273-4742. [CrossRef] [PubMed]

15. Massari, S.; Ruberti, M. Rare earth elements as critical raw materials: Focus on international markets and future strategies. Res. Policy 2013, 38, 36-43. [CrossRef]

16. Bünzli, J.-C.G.; McGill, I. Rare Earth Elements. In Ullmann's Encyclopedia of Industrial Chemistry, 7th ed.; Wiley-VCH Verlag 419 GmbH \& Co. KGaA: Weinheim, Germany, 2018; pp. 1-53.

17. Balzani, V.; Ceroni, P.; Juris, A. Photochemistry and Photophysics: Concepts, Research, Applications; Wiley-VCH Verlag GmbH \& Co. KGaA: Weinheim, Germany, 2014.

18. Utochnikova, V.V. The use of luminescent spectroscopy to obtain information about the composition and the structure of lanthanide coordination compounds. Coord. Chem. Rev. 2019, 398, 113006. [CrossRef]

19. Bünzli, J.-C.G.; Pecharsky, V.K. Handbook on the Physics and Chemistry of Rare Earths, 1st ed.; Elsevier: Amsterdam, The Netherlands, 2021.

20. Bünzli, J.-C.G. Lanthanide Photonics: Shaping the Nanoworld. Trends Chem. 2019, 1, 751-762. [CrossRef]

21. Ning, Y.; Zhu, M.; Zhang, J.-L. Near-infrared (NIR) lanthanide molecular probes for bioimaging and biosensing. Coord. Chem. Rev. 2019, 399, 213028. [CrossRef]

22. Zhou, J.; Liu, Q.; Feng, W.; Sun, Y.; Li, F. Upconversion Luminescent Materials: Advances and Applications. Chem. Rev. 2015, 115, 395-465. [CrossRef]

23. Cui, Y.; Chen, B.; Qian, G. Lanthanide metal-organic frameworks light-emitting for luminescent sensing and applications. Coordin. Chem. Rev. 2014, 273-274, 76-86. [CrossRef]

24. Bünzli, J.-C.G. On the design of highly luminescent lanthanide complexes. Coord. Chem. Rev. 2015, 293-294, 19-47. [CrossRef]

25. Binnemans, K. Lanthanide-Based Luminescent Hybrid Materials. Chem. Rev. 2009, 109, 4283-4374. [CrossRef]

26. Feng, J.; Zhang, H. Hybrid materials based on lanthanide organic complexes: A review. Chem. Soc. Rev. 2013, 42, 387-410. [CrossRef]

27. Brites, C.D.S.; Balabhadra, S.; Carlos, L.D. Lanthanide-Based Thermometers: At the Cutting-Edge of Luminescence Ther-mometry. Adv. Optical Mater 1002, 7.

28. Hehlen, M.P.; Sheik-Bahae, M.; Epstein, R.I. Solid-state Optical Refrigeration. In Handbook on the Physics and Chemistry of Rare Earths; Bünzli, J.-C.G., Pecharsky, V.K., Eds.; Elsevier Science B. V.: Amsterdam, The Netherlands, 2014; Volume 45, pp. 179-260.

29. Aguila, D.; Roubeau, O.; Aromi, G. Designed Polynuclear Lanthanide Complexes for Quantum Information Processing. Dalton Trans. 2021. accepted manuscript (August 2021). [CrossRef] 
30. Zhang, H.; Chen, Z.-H.; Liu, X.; Zhang, F. A mini-review on recent progress of new sensitizers for luminescence of lanthanide doped nanomaterials. Nano Res. 2020, 13, 1795-1809. [CrossRef]

31. Mikhalyova, E.A.; Pavlishchuk, V.V. Modern approaches to the tuning of the lanthanide $(3+)$ coordination compound lumi-nescent characteristics: A review. Theor. Exp. Chem. 2019, 55, 293-315. [CrossRef]

32. Carrasco, I.; Piccinelli, F.; Bettinelli, M. Luminescence of Tb-based materials doped with Eu3+: Case studies for energy transfer processes. J. Lumin. 2017, 189, 71-77. [CrossRef]

33. Jaiswal, S.R.; Nagpure, P.A.; Omanwar, S.K. Improvement of quantum efficiency through Gd 3+ to Eu 3+ energy transfer in YF 3 phosphor. Luminescence 2021, 36, 1395-1401. [CrossRef]

34. D'Aléo, A.; Pointillart, F.; Ouahab, L.; Andraud, C.; Maury, O. Charge transfer excited states sensitization of lanthanide emitting from the visible to the near-infra-red. Coord. Chem. Rev. 2012, 256, 1604-1620. [CrossRef]

35. Joos, J.J.; Neefjes, I.; Sejio, L.; Barandiaran, Z. Charge transfer from Eu2+ to trivalent lanthanide co-dopants: Systematic be-haviour across the series. J. Chem. Phys. 2021, 154, 064704. [CrossRef]

36. Sapsford, K.E.; Berti, L.; Medintz, I.L. Materials for Fluorescence Resonance Energy Transfer Analysis: Beyond Traditional Donor-Acceptor Combinations. Angew. Chem. Int. Ed. 2006, 45, 4562-4589. [CrossRef]

37. Wei, C.; Ma, L.; Wei, H.; Liu, Z.; Bian, Z.; Huang, C. Advances in luminescent lanthanide complexes and applications. Sci. China Ser. E Technol. Sci. 2018, 61, 1265-1285. [CrossRef]

38. Liu, L.; Lu, H.; Wang, H.; Bei, Y.; Feng, S. Luminescent organo-polysiloxanes containing complexed lanthanide ions. Appl. Organomet. Chem. 2009, 23, 429-433. [CrossRef]

39. Carniato, F.; Gatti, G.; Bisio, C. An overview of the recent synthesis and functionalization methods of saponite clay. New J. Chem. 2020, 44, 9969-9980. [CrossRef]

40. Marchesi, S.; Carniato, F.; Guidotti, M.; Botta, M.; Marchese, L.; Bisio, C. Synthetic saponite clays as promising solids for lan-thanide ion recovery. New J. Chem. 2020, 44, 10033-10041. [CrossRef]

41. Marchesi, S.; Carniato, F.; Bisio, C.; Tei, L.; Marchese, L.; Botta, M. Novel paramagnetic clays obtained through intercalation of Gd3+-complexes. Dalton Trans. 2018, 47, 7896-7904. [CrossRef]

42. Lalli, D.; Marchesi, S.; Carniato, F.; Bisio, C.; Tei, L.; Marchese, L.; Botta, M. Combination of solid-state NMR and 1H NMR relaxometry for the study of intercalated saponite clays with the macrocyclic derivatives of Gd(iii) and Y(iii). Dalton Trans. 2020, 49, 6566-6571. [CrossRef]

43. Marchesi, S.; Guidotti, M.; Marchese, L.; Evangelisti, C.; Carniato, F.; Bisio, C. Bifunctional europium(III) and niobi-um(V)containing Saponite Clays for the Simultaneous Optical Detection and Catalytic Oxidative Abatement of Blister Chemical Warfare Agents. Chem.-Eur. J. 2021, 27, 4723-4730. [CrossRef]

44. Marchesi, S.; Bisio, C.; Carniato, F. Novel light-emitting clays with structural Tb3+ and Eu3+ for chromate anion detection. RSC Adv. 2020, 10, 29765-29771. [CrossRef]

45. Marchesi, S.; Bisio, C.; Lalli, D.; Marchese, L.; Platas-Iglesias, C.; Carniato, F. Bifunctional Paramagnetic and Luminescent Clays Obtained by Incorporation of Gd3+ and Eu3+ Ions in the Saponite Framework. Inorg. Chem. 2021, 60, 10749-10756. [CrossRef]

46. Guo, X.; Fu, L.; Zhang, H.; Carlos, L.D.; Peng, C.; Guo, J.; Yu, J.; Deng, R.; Sun, L. Incorporation of luminescent lanthanide complex inside the channels of organically modified mesoporous silica via template-ion exchange method. New J. Chem. 2005, 29, 1351-1358. [CrossRef]

47. Maouche, R.; Belaid, S.; Benmerad, B.; Bouacida, S.; Daiguebonne, C.; Suffren, Y.; Freslon, S.; Bernot, K.; Guillou, O. Highly Luminescent Europium-Based Heteroleptic Coordination Polymers with Phenantroline and Glutarate Ligands. Inorg. Chem. 2021, 60, 3707-3718. [CrossRef] [PubMed]

48. Liu, J.; Sun, W.; Liu, Z. White-light emitting materials with tunable luminescence based on steady Eu(iii) doping of Tb(iii) metal-organic frameworks. RSC Adv. 2016, 6, 25689-25694. [CrossRef]

49. Liu, X.; Hu, Y.; Wang, B.; Su, Z. Synthesis and fluorescent properties of europium-polymer complexes containing 1,10phenanthroline. Synth. Met. 2009, 159, 1557-1562. [CrossRef]

50. Andrews, D.L. Mechanistic principles and applications of resonance energy transfer. Can. J. Chem. 2008, 86, 855-870. [CrossRef]

51. Teng, Q.; Huynh, H.V. A unified ligand electronic parameter based on 13C NMR spectroscopy of N-heterocyclic carbene complexes. Dalton Trans. 2016, 46, 614-627. [CrossRef] [PubMed]

52. Binnemans, K. Interpretation of europium(III) spectra. Coord. Chem. Rev. 2015, 295, 1-45. [CrossRef]

53. Wegh, R.; Meijerinkx, A. First Observation of Visible Luminescence from Trivalent Gadolinium. Acta Phys. Pol. A 1996, 90, 333-337. [CrossRef]

54. Zhang, P.; Wang, Y.; Liu, H.; Chen, Y. Preparation and luminescence of europium(iii) terpyridine complex-bridged polysilsesquioxanes. J. Mater. Chem. 2011, 21, 18462-18466. [CrossRef]

55. Tang, S.; Babai, A.; Mudring, A.-V. Europium-Based Ionic Liquids as Luminescent Soft Materials. Angew. Chem. Int. Ed. 2008, 47, 7631-7634. [CrossRef]

56. Marchesi, S.; Carniato, F.; Boccaleri, E. Synthesis and characterisation of a novel europium(iii)-containing heptaisobutyl-POSS. New J. Chem. 2014, 38, 2480-2485. [CrossRef]

57. Marchesi, S.; Carniato, F.; Marchese, L.; Boccaleri, E. Luminescent Mesoporous Silica Built through Self-Assembly of Polyhedral Oligomeric Silsesquioxane and Europium(III) Ions. ChemPlusChem 2015, 80, 915-918. [CrossRef] [PubMed] 
58. Marchesi, S.; Bisio, C.; Boccaleri, E.; Carniato, F. A Luminescent Polysilsesquioxane Obtained by Self-Condensation of Anionic Polyhedral Oligomeric Silsequioxanes (POSS) and Europium(III) Ions. ChemPlusChem 2020, 85, 176-182. [CrossRef]

59. Sahu, I.P.; Bisen, D.; Tamrakar, R.K.; Murthy, K.; Mohapatra, M. Luminescence studies on the europium doped strontium metasilicate phosphor prepared by solid state reaction method. J. Sci. Adv. Mater. Devices 2017, 2, 59-68. [CrossRef]

60. Cucinotta, F.; Carniato, F.; Devaux, A.; De Cola, L.; Marchese, L. Efficient Photoinduced Energy Transfer in a Newly Devel-oped Hybrid SBA-15 Photonic Antenna. Chem. Eur. J. 2012, 18, 15310-15315. [CrossRef]

61. Olivero, F.; Carniato, F.; Bisio, C.; Marchese, L. Promotion of Förster Resonance Energy Transfer in a Saponite Clay Containing Luminescent Polyhedral Oligomeric Silsesquioxane and Rhodamine Dye. Chem.-Asian J. 2014, 9, 158-165. [CrossRef]

62. Aime, S.; Botta, M.; Terreno, E. Gd(III)-BASED CONTRAST AGENTS FOR MRI. Insights Imaging Bioinorg. Chem. 2005, 57, 173-237. [CrossRef]

63. Hermann, P.; Kotek, J.; Kubicek, V.; Lukes, I. Gadolinium(iii) complexes as MRI contrast agents: Ligand design and properties of the complexes. Dalton Trans. 2008, 21, 3027-3047. [CrossRef] [PubMed] 